\title{
Validez de la prueba de adenosina deaminasa y del recuento diferencial de leucocitos para el diagnóstico de tuberculosis pleural
}

\author{
Validity of both adenosine deaminase test and differential cell count for pleural \\ tuberculosis diagnosis
}

\author{
Jhonatan Boris Quiñones-Silva ${ }^{1,2}$, César Héctor Gonzalo Ramírez-Yépez ${ }^{1,2}$, \\ Américo Peña-Oscuvilca ${ }^{1,2}$, Efraín Estrada-Choque ${ }^{1,3}$ \\ ${ }^{1}$ Facultad de Medicina Humana, Universidad Nacional José Faustino Sánchez Carrión. Huacho, Perú. \\ ${ }^{2}$ Sociedad Científica Huachana de Estudiantes de Medicina. Huacho, Perú. \\ ${ }^{3}$ Hospital Gustavo Lanatta Luján, Ministerio de Salud. Huacho, Perú.
}

\section{Resumen}

Objetivo: Determinar la validez de la prueba de adenosina deaminasa (ADA) y el recuento diferencial de leucocitos para el diagnóstico de tuberculosis pleural. Diseño: Estudio retrospectivo de precisión diagnóstica. Institución: Hospital Regional de Huacho, Ministerio de Salud, Huacho, Perú. Material: Historias clínicas de pacientes con prueba de ADA en líquido pleural. Intervenciones: Estudio de historias clínicas de pacientes a quienes se les realizó la prueba de ADA en líquido pleural, desde enero 2005 hasta diciembre 2007. El valor de la prueba de ADA y el recuento diferencial de leucocitos fueron analizados mediante la curva ROC. La sensibilidad (Se), especificidad (Sp), valores predictivos (VPP y VPN) y los cocientes de verosimilitud (CVP y CVN) fueron hallados para cada variable. Principales medidas de resultados: Punto de corte para la prueba de ADA para el diagnóstico de tuberculosis pleural. Resultados: La tuberculosis fue culpable de la mayoría de los derrames pleurales; 47,5 fue el punto de corte hallado para la prueba de ADA, con Se y Sp de $73 \%$ y $90 \%$, respectivamente (IC 95\%: 0,74 $0,95 ; p<0,001)$. La curva ROC para el recuento diferencial de leucocitos dio un punto de corte de 0,725; con Se y Sp de 60,9 y 64,7, respectivamente (IC 95\%: $0,47-0,79 ; p=0,129)$. Conclusiones: El mejor punto de corte discriminatorio para el diagnóstico de tuberculosis pleural fue 47,5 UI/L. Esta prueba sirve más para confirmar el diagnóstico que para descartar la enfermedad. Además, un mayor porcentaje de linfocitos en el recuento diferencial de leucocitos $(>72 \%)$ incrementaría la sospecha de tuberculosis pleural.

Palabras clave: Tuberculosis pleural; adenosindesaminasa; recuento de leucocitos diferencial.

\begin{abstract}
Objective: To determine the validity of both adenosine diaminase (ADA) test and differential cell count for pleural tuberculosis diagnosis. Design: Retrospective study for diagnosis precision. Setting: Huacho Hospital, Ministerio de Salud, Huacho, Peru. Material: Medical records of patients with ADA test in pleural fluid. Interventions: Medical records study of patients with ADA test in pleural effusion performed from January 2005 through December 2007. ADA test values and differential cell counts were analyzed by ROC curve. Sensitivity (Se), specificity (Sp), predictive values (VPP and VPN) and likehood ratios (LRP and LRN) were found for each variable. Main outcome measures: ADA test cut-off point for pleural tuberculosis diagnosis. Results: Tuberculosis was responsible for most cases of pleural effusion; $47,5 \mathrm{UI} / \mathrm{L}$ was the cut-off point by ROC curve, with $\mathrm{Se}$ and Sp $73 \%$ and $90 \%$, respectively (IC 95\%: $0,74-0,95 ; p<0,001$ ). The ROC curve for differential cell count cut-off point was 0,725, with Se and Sp 60,9 and 64,7, respectively (IC 95\%: 0,47 - 0,79; $p=0,129$ ). Conclusions: The best cut-off point for pleural tuberculosis diagnosis was $47,5 \mathrm{UI} / \mathrm{L}$. This test worked better to confirm the disease rather than ruling it out. Thus, a larger percentage of lymphocytes in the differential cell count $(>72 \%)$ will increase the suspicious of pleural tuberculosis.
\end{abstract}

Key words: Tuberculosis, pleural; adenosine deaminase; leukocyte count.

\section{INTRODUCCIÓN}

La prueba de adenosina deaminasa (ADA, del inglés: adenosine deaminase) es un examen muy usado para el diagnóstico de tuberculosis extrapulmonar. Esta prueba colorimétrica se basa en la cuantificación del amonio que surge como resultado de la acción de la enzima adenosina deaminasa ${ }^{(1)}$.

La prueba de ADA en líquido pleural es un examen diagnóstico útil en pacientes con efusión pleural de etiología a incierta, sobre todo en lugares donde existe una prevalencia de tuberculosis alta, logrando diferenciar esta de otras causas de derrame pleural, como neumonías, neoplasias, colagenopatías, lo cual nos permite tomar decisiones terapéuticas tempranas.

La determinación de los parámetros de la prueba de $\mathrm{ADA}$, al igual que cualquier otra prueba diagnóstica, está determinada por la prevalencia de la enfermedad en la población estudiada, el diseño y la metodología del estudio. Lo que lleva a que existan diferentes valores discriminatorios (puntos de corte) para esta prueba diagnóstica ${ }^{(2)}$. En nuestro país, el punto de corte es $45 \mathrm{UI} / \mathrm{L}$, no habiendo estudio nacional que lo sustente.
Por otra parte, la utilidad del recuento diferencial de leucocitos (linfocitos y neutrófilos) en el líquido pleural incrementa la precisión diagnóstica de tuberculosis pleural cuando existe un mayor infiltrado linfocitario ${ }^{(3)}$. De esta manera, se puede observar que la presunción diagnóstica de tuberculosis pleural se ve favorecida si además de la obtención del valor de la prueba de ADA se encuentra un mayor infiltrado linfocitario.

Así, la ausencia de algún estudio publicado en el Perú mediante análisis estadísticos adecuados sobre la determinación de la utilidad de esta prueba 
diagnóstica, y por la alta prevalencia de tuberculosis en nuestro país, conduce a la ejecución de un estudio cuyo objetivo fue determinar la validez y eficacia de la prueba de ADA y del recuento diferencial de leucocitos, mediante parámetros como sensibilidad, especificidad, los valores predictivos, además del punto de corte (UI/L) más adecuado para la prueba.

\section{MÉTODOS}

Se realizó un estudio retrospectivo de precisión diagnóstica en el Hospital Regional de Huacho. Se obtuvo el acceso de las historias clínicas de los pacientes a quienes se les realizó la prueba de ADA en el líquido pleural, desde enero de 2005 hasta diciembre de 2007. Todos los exámenes realizados fueron analizados en el Laboratorio central del mismo hospital, según el método estándar para la determinación cuantitativa de adenosina deaminasa.

Para la determinación del punto de corte para la prueba de ADA y el recuento diferencial de leucocitos, se consideró la máxima sumatoria de la sensibilidad y especificidad mediante la curva de características operativas del receptor (ROC, del inglés: receiver operating characteristic). Una vez obtenido el punto de corte, se construyó una tabla de contingencia, mediante la cual se determinó el valor predictivo positivo $(\mathrm{VP}+)$, valor predictivo negativo (VP-), el cociente de verosimilitud positivo $(\mathrm{CV}+)$ y el cociente de verosimilitud negativo ( $\mathrm{CV}-$ ) de las mismas.

El diagnóstico de tuberculosis pleural (TP) se estableció si se encontraba en la historia clínica al menos uno de los siguientes criterios: a) identificación del bacilo de Koch (BK) en el líquido o biopsia pleural, cultivo o por la observación de granulomas en el estudio histológico; b) cultivo de esputo en presencia de evidencia clínica o radiológica de tuberculosis, en ausencia de otra causa obvia asociada al derrame pleural; o c) evidencia clínica o radiológica de tuberculosis en ausencia de otra causa obvia asociada a derrame pleural y con una respuesta positiva a la terapia antituberculosa. El diagnóstico de las demás entidades se basó en los criterios respectivos para su confirmación.

Aquellas historias clínicas que no consignaron un diagnóstico definitivo o carecían de datos de acuerdo a lo requerido en el estudio fueron excluidas del estudio. Los datos obtenidos fueron procesados y analizados mediante el paquete informático SPSS 15,0 para Windows.

\section{RESULTADOS}

De las 265 historias revisadas, 187 quedaron excluidas debido a que carecían de algunos datos requeridos para el estudio, además de no cumplir con los criterios para el diagnóstico de TP. De los 74 casos finalmente incluidos, 42 (56,8\%) fueron varones; y la edad media fue 44,6 años, con una desviación típica de 21,1, en un rango de 14 a 87 años.

Las entidades clínicas según su presentación por sexo son informadas en la tabla 1;

Tabla 1. Entidades clínicas informadas en el estudio según sexo.

\begin{tabular}{|c|c|c|c|c|c|}
\hline \multirow[b]{2}{*}{ Diagnóstico } & \multicolumn{2}{|c|}{ Varones } & \multicolumn{2}{|c|}{ Mujeres } & \multirow[b]{2}{*}{ Total } \\
\hline & $\mathrm{n}$ & $\%$ & $\mathrm{n}$ & $\%$ & \\
\hline Tuberculosis pleural & 34 & 81,0 & 19 & 59,4 & 53 \\
\hline Paraneumónico & 5 & 11,9 & 2 & 6,3 & 7 \\
\hline Insuficiencia cardíaca congestiva & 2 & 4,8 & 4 & 12,5 & 6 \\
\hline Neoplasia maligna & 1 & 2,4 & 5 & 15,6 & 6 \\
\hline Lupus eritematoso sistémico & 0 & 0,0 & 2 & 6,3 & 2 \\
\hline Total & 42 & 100,0 & 32 & 100,0 & 74 \\
\hline
\end{tabular}

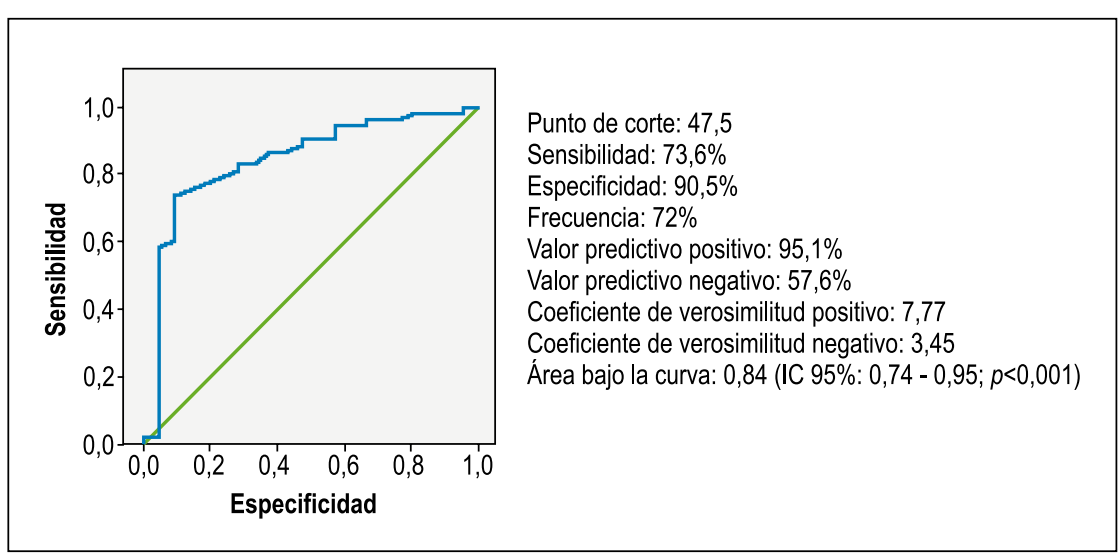

Figura 1. Curva ROC y demás parámetros de la prueba de adenosina deaminasa. la enfermedad de mayor prevalencia en la muestra fue tuberculosis pleural, que fue diagnosticada en $53(71,6 \%)$ pacientes y de ellos 34 (81\%) fueron varones.

El punto de corte calculado de adenosina deaminasa mediante la curva ROC fue 47,5 UI/L, con una sensibilidad de $73 \%$ y especificidad de $90 \%$ (figura 1 ), cuya área bajo la curva fue 0,84 (IC 95\%: $0,74-0,95 ; p<0,001)$. Con estos datos, se determinó el valor predictivo positivo y negativo, que fueron $95,1 \%$ y $57,6 \%$, respectivamente. Del mismo modo, los coeficientes de verosimilitud positivo y negativo fueron 7,77 y 3,45 , respectivamente.

A once pacientes no se les practicó el examen de recuento de linfocitos y neutrófilos, por lo que fueron excluidos del análisis. Para la relación entre linfocitos y el total de leucocitos, el punto de corte determinado fue 0,725 (IC 95\%: 0,47 $0,79 ; p=0,129)$. La curva ROC de este indicador se encuentra en la figura 2. 


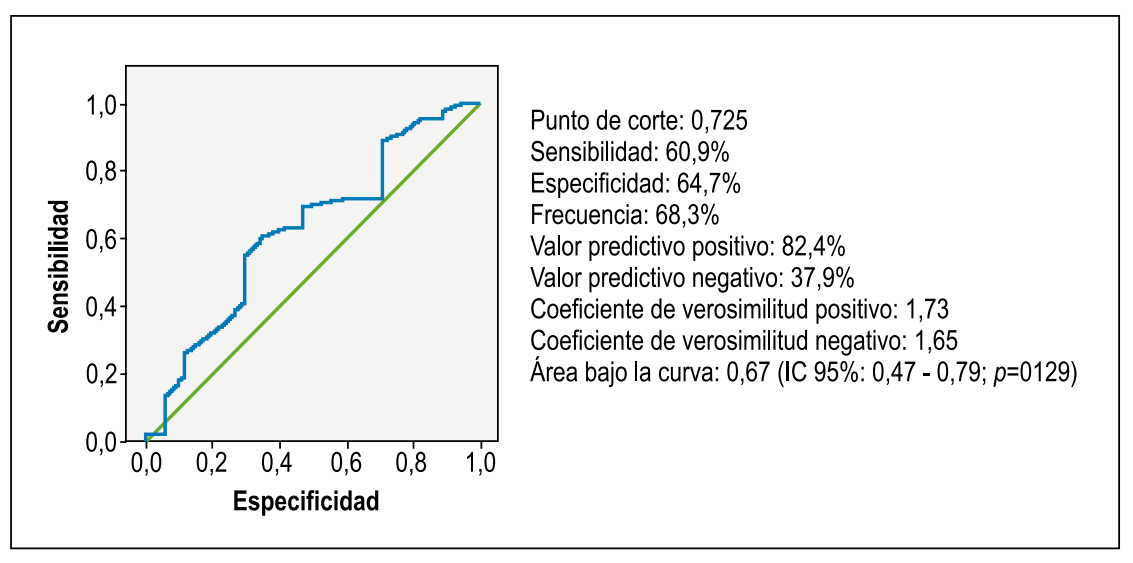

Figura 2. Curva ROC y demás parámetros del recuento diferencial leucocitario.

\section{DISCUSIÓN}

Las comunicaciones estiman que la forma de presentación extrapulmonar de la tuberculosis representa el 10 por ciento del total, siendo la tuberculosis pleural la segunda causa más frecuente, precedida solo por la tuberculosis linfonodular ${ }^{(2,4)}$. En este estudio, del total de pacientes que presentaron derrame pleural, la tuberculosis fue responsable de más de $70 \%$, mientras que el resto de los casos fueron procesos paraneumónicos, insuficiencia cardiaca, neoplasias y lupus eritematoso sistémico.

El diagnóstico de esta patología está asociado clásicamente al hallazgo bacteriológico del bacilo en el líquido pleural; desafortunadamente, los resultados de la tinción para la búsqueda del Mycobacterium tuberculosis son usualmente negativos y el cultivo del mismo es positivo en menos de 25 por ciento de los casos (5). Por otro lado, una muestra de biopsia pleural demostrará pleuritis granulomato- sa en 80 por ciento de los pacientes con derrame pleural de origen tuberculoso; y cuando se combina el cultivo de la biopsia con el examen histológico, el diagnóstico puede establecerse en aproximadamente 90 por ciento de los casos ${ }^{(5)}$. No obstante, muchos centros asistenciales no cuentan con personal entrenado ni con equipos necesarios para la realización de estos procedimientos; además, la carencia económica de estos pacientes limita la realización de dichas pruebas; por lo que el diagnóstico etiológico se basa en su mayoría de veces en el examen físico y radiológico del paciente y el examen bioquímico del líquido pleural obtenido por toracocentesis, entre ellos la prueba de $\operatorname{ADA}^{(6)}$.

En relación a la prueba de ADA como diagnóstica de tuberculosis pleural, diversos autores han encontrado diferentes puntos de corte, que varían desde 30 hasta $100 \mathrm{UI} / \mathrm{L}$ (tabla 2) ${ }^{(7-24)}$, dependiendo esto del grupo de pacientes que formaron parte del estudio, la población cuya epidemiología del Mycobacterium es distinta y los criterios tomados en cuenta para el diagnóstico definitivo de las diferentes enfermedades que ocasionaron el derra-

Tabla 2. Estudios realizados a lo largo del tiempo sobre el valor de la prueba de ADA y sus principales parámetros.

\begin{tabular}{|c|c|c|c|c|c|c|c|c|}
\hline Estudio & País & Año & $\begin{array}{c}\mathrm{N}^{\circ} \text { de } \\
\text { pacientes }\end{array}$ & $\begin{array}{l}\text { Punto } \\
\text { de corte }\end{array}$ & Sensibilidad & Especificidad & $\begin{array}{l}\text { Valor predictivo } \\
\text { positivo }\end{array}$ & $\begin{array}{c}\text { Valor predictivo } \\
\text { negativo }\end{array}$ \\
\hline Piras y col ${ }^{(7)}$ & Italia & 1978 & 54 & 30 & 100 & 100 & -- & -- \\
\hline Ocaña y col ${ }^{(8)}$ & España & 1983 & 182 & 45 & 100 & 97 & -- & -- \\
\hline Segura y col ${ }^{(9)}$ & España & 1989 & 600 & 71 & 100 & 92 & -- & -- \\
\hline Valdes y col (10) & España & 1993 & 405 & 47 & 100 & 95 & 85 & 100 \\
\hline De Olivera y col (11) & Brasil & 1994 & 276 & 40 & 91 & 88 & -- & -- \\
\hline Burgess y col ${ }^{(12)}$ & Sudáfrica & 1995 & 462 & 50 & 90 & 89 & 81 & 94 \\
\hline Valdes y col ${ }^{(13)}$ & España & 1996 & 350 & 47 & 100 & 91 & -- & -- \\
\hline Villena y col (14) & España & 1996 & 228 & 33 & 90 & 85 & -- & -- \\
\hline Chalhoub y col ${ }^{(15)}$ & Brasil & 1996 & 221 & 40 & 93,3 & 93,5 & 97,2 & 85,3 \\
\hline Pérez-Rodríguez y col (16) & España & 1999 & 103 & 40 & 89 & 92 & 80 & 95,8 \\
\hline Villegas y col (17) & Colombia & 2000 & 140 & 45.5 & 88 & 86 & -- & -- \\
\hline Sharma y col ${ }^{(18)}$ & India & 2001 & 75 & 35 & 83 & 67 & -- & -- \\
\hline Sharma y col ${ }^{(18)}$ & India & 2001 & 75 & 100 & 40 & 100 & -- & -- \\
\hline Reechaipichitkul y col (19) & Tailandia & 2001 & 132 & 48 & 80 & 81 & 71,4 & 86,8 \\
\hline Lima y col (20) & Brasil & 2003 & 45 & 40 & 68 & 72 & 57,9 & 80,8 \\
\hline Diacon y col (21) & Sudáfrica & 2003 & 51 & 50 & 95 & 89 & 97 & 80 \\
\hline Tahhan y col (22) & Turquía & 2003 & 62 & 40 & 91 & 89 & -- & -- \\
\hline Kaisemann y col (23) & Brasil & 2004 & 111 & 35 & 92,8 & 96,4 & -- & -- \\
\hline Neves y col (24) & Brasil & 2004 & 215 & 39 & 94,2 & 82,9 & -- & -- \\
\hline Presente estudio & Perú & 2010 & 74 & 47.5 & 73 & 90 & 95,1 & 57,6 \\
\hline
\end{tabular}


me pleural. No obstante las diferencias encontradas en los estudios, los autores no dudan su utilidad; incluso algunos indican que el rendimiento diagnóstico de ADA parece ser lo suficientemente alto para evitar biopsia pleural en pacientes reacios a sufrir procedimientos invasivos, especialmente en regiones que tienen una prevalencia alta de M. tuberculosis ${ }^{(25)}$.

En un metaanálisis de estudios realizados en Brasil, se concluyó que la prueba de ADA tiene una alta precisión en el diagnóstico de tuberculosis pleural y debería ser usada como examen de rutina en su investigación ${ }^{(26)}$. Otro metaanálisis publicado recientemente encontró que no se afectaba sustancialmente la precisión de la prueba al comparar estudios que usaban el método Giusti versus el no Giusti, si es que estaban incluidos o no los trasudados en el grupo de no tuberculosos, o si es que el estudio fue prospectivo o retrospectivo ${ }^{(27)}$.

El estudio de Piras y col ${ }^{(7)}$, no obstante ser pionero sobre la materia, fue duramente criticado, puesto que su muestra solo incluyó a pacientes sanos y tuberculosos, excluyendo a otras causas de derrame pleural. Esto hizo que los valores de sensibilidad y especificidad fuesen sumamente elevados, tergiversando su verdadera utilidad como prueba diagnóstica. Posteriormente, nuevas investigaciones señalaban ser cautelosos ante los resultados de la prueba por la presencia de muchos falsos positivos, y recomendaban complementar el estudio con pruebas invasivas, como una biopsia pleural ${ }^{(28)}$.

El punto de corte obtenido en este estudio $(47,5 \mathrm{UI} / \mathrm{L})$ no se aleja mucho del usado en el laboratorio central del Hospital Regional de Huacho (45 UI/L), aunque cabe recalcar que no se realizó estudio previo en la región para consignar este valor discriminatorio. El punto de corte de $45 \mathrm{UI} / \mathrm{L}$, usado como referencia en los hospitales del Perú para el diagnóstico de tuberculosis pleural, según Ortiz-Sánchez ${ }^{(29)}$, se basa en un estudio realizado por Ocaña y col ${ }^{(8)}$, en Barcelona, España. Hecho contraproducente, entendiendo que no se debe extrapolar estos datos, sobre todo cuando la prevalencia es distinta en ambas poblaciones estudiadas.
Además, estudios peruanos realizados con respecto a este tema ${ }^{(29,30)}$ hallan el punto de corte con limitaciones metodológicas, ya que no analizan esta variable mediante la curva ROC, que es la prueba estadística que determina el mejor punto de corte, a través de la mejor relación entre la sensibilidad y la especificidad ${ }^{(31)}$.

La especificidad alta y sensibilidad baja encontrados en el estudio nos indican que esta prueba sirve más como una prueba para diagnosticar tuberculosis pleural, una vez se tenga la sospecha clínica o radiológica, que para descartar su presencia. De esta forma, un resultado positivo sería mejor aprovechado por el clínico, más que un resultado negativo.

Por otra parte, habiendo sido demostrada la utilidad del recuento diferencial de leucocitos para el diagnóstico de TP (32), en nuestro estudio el mejor valor discriminatorio para el diagnóstico de esta patología fue 0,725 , lo que indica que, si en el recuento diferencial de células existe más de $72 \%$ de linfocitos, es más probable que el diagnóstico sea tuberculosis pleural. Aunque, este valor no es estadísticamente significativo $(p=0,129)$. De igual modo, el resultado de los parámetros que determinan su utilidad no es muy alentador.

No obstante, se debe ser cauteloso con estos resultados, ya que quizá un tamaño muestral mayor pudo haber encontrado una diferencia estadísticamente significativa, por lo que el número de sujetos es una limitación del estudio.

Se concluye que 47,5 UI/L es el mejor punto de corte discriminatorio para el diagnóstico de tuberculosis pleural y que esta prueba sirve más como una prueba para confirmar el diagnóstico, una vez sospechada la enfermedad, que para descartarla. Además, un mayor porcentaje de linfocitos en el recuento diferencial de leucocitos (>72\%) incrementaría la sospecha de efusión pleural.

Finalmente, se recomienda que se elabore trabajos de investigación de la misma naturaleza en las diferentes regiones, para así encontrar su verdadera utilidad en cada una de estas.
El presente trabajo ganó en el 2008 el concurso de trabajos científicos organzado por la Sociedad Peruana de Estudiantes de Medicina (SOCIPEM) realizada en Lima, en la "Categoría Clínico-Quirúrgicas".

\section{REFERENCIAS BIBLIOGRÁFICAS}

1. Slaats EH, Asberg EG, van Keimpema AR, Kruijswijk $H$. A continuous method for the estimation of adenosine deaminase catalytic concentration in pleural effusions with a Hitachi 705 discrete analyser. J Clin Chem Clin Biochem. 1985;23(10):677-82.

2. Gopi A, Madhavan SM, Sharma SK, Sahn SA. Diagnosis and treatment of tuberculous pleural effusion in 2006. Chest. 2007;131(3):880-9.

3. Burgess LJ, Maritz FJ, Le Roux I, Taljaard JJ. Combined use of pleural adenosine deaminase with lymphocyte/neutrophil ratio. Increased specificity for the diagnosis of tuberculous pleuritis. Chest. 1996;109(2):414-9.

4. Golden MP, Vikram HR. Extrapulmonary tuberculosis: an overview. Am Fam Physician. 2005;72:1761-8.

5. Laniado-Laborín R. Adenosine deaminase in the diagnosis of tuberculous pleural effusion. Is it really an ideal test? A word of caution [editorial]. Chest. 2005;127(2):417-8.

6. Estrada-Choque E, Moscol S. Progresos en el diagnóstico de patología pleural en el Hospital "Gustavo Lanatta Luján" Huacho. Rev Méd IPSS. 1994;3(2):7-14.

7. Piras MA, Gakis C, Budroni M, Andreoni G. Adenosine deaminase activity in pleural effusions: an aid to differential diagnosis. BMJ. 1978;2:1751-2.

8. Ocaña I, Martinez-Vasquez JM, Segura RM, Fernandez-De-Sevilla T, Capdevila JA. Adenosine deaminase in pleural fluids. Test for diagnosis of tuberculosis pleural effusion. Chest. 1983;84(1):51-3.

9. Segura RM, Pascaual C, Ocaña I, MartínezVázquez JM, Ribera E, Ruiz I, et al. Adenosine deaminase in body fluids: a useful diagnostic tool in tuberculosis. Clin Biochem. 1989;22:141-8.

10. Valdes L, San Jose E, Alvarez D, Sarandeses A, Pose A, Chomón B, et al. Diagnosis of tuberculous pleurisy using the biologic parameters adenosine deaminase, lysozyme, and interferon gamma. Chest. 1993;103:458-65.

11. De Olivera HG, Rossatto ER, Prolla JC. Pleural fluid adenosine deaminase and lymphocyte proportion: clinical usefulness in the diagnosis of tuberculosis. Cytopathology. 1994;5:27-32.

12. Burguess LJ, Maritz FJ, Le Roux I, Taljaard JJ. Use of adenosine deaminase as a diagnostic tool for tuberculosis pleurisy. Thorax. 1995;50:672-4.

13. Valdes L, San José E, Alvarez D, Valle JM. Adenosine deaminase (ADA) isoenzyme analysis in pleural effusions: diagnostic role and relevance to the origin of increased ADA in 
tuberculous pleurisy. Eur Respir J. 1996;9:74751.

14. Villena V, Navarro-Gonzales JA, García-Benayas C, Manzanos JA, Echave J, López-Encuentra A, et al. Rapid automated determination of adenosine deaminase and lysozyme for differentiating tuberculous and nontuberculous pleural effusions. Clin Chem. 1996;42:218-21.

15. Chalhoub M, Cruz AA, Marcilio C, Netto MB. Valor da determinação da atividade da adenosina deaminase (ADA) no diagnóstico diferencial dos derrames pleurais. Rev Assoc Med Bras. 1996;42(3):139-46.

16. Pérez-Rodríguez E, Pérez Walton IJ, Sanchez Hernández JJ, Pallarés E, Rubi J, Jimenez Castro $D$, et al. ADA1/ADAp ratio in pleural tuberculosis: an excellent diagnostic parameter in pleural fluid. Respir Med. 1999;9:816-21.

17. Villegas MV, Labrada LA, Saravia NG. Evaluation of polymerase chain reaction, adenosine deaminase, and interferon-gamma in pleural fluid for the differential diagnosis of pleural tuberculosis. Chest. 2000;118:1355-64.

18. Sharma SK, Suresh V, Mohan A, Kaur P, Saha P, Kumar A, et al. A prospective study of sensitivity and specificity of adenosine deaminase estimation in the diagnosis of tuberculosis pleural effusion. Indian J Chest Dis Allied Sci. 2001;43:149-55.

19. Reechaipichitkul W, Kawamatawong T, Teerajetgul Y, Patjanasoontorn B. Diagnostic role of pleural fluid adenosine deaminase in tuberculous pleural effusion. Southeast Asian J Trop Med Public Health. 2001;32:383-9.
20. Lima DM, Colares JK, da Fonseca BA. Combined use of the polymerase chain reaction and detection of adenosine deaminase activity on pleural fluid improves the rate of diagnosis of pleural tuberculosis. Chest. 2003;124:909-14.

21. Diacon AH, Van de Wal BW, Wyser C, Smedema JP, Bezuidenhout J, Bolliger CT, et al. Diagnostic tools in tuberculous pleurisy: a direct comparative study. Eur Respir J. 2003;22:589-91.

22. Tahhan M, Ugurman F, Gozu A, Akkalyoncu B, Samurkasoglu B. Tumour necrosis factor-alpha in comparison to adenosine deaminase in tuberculous pleuritis. Respiration. 2003;70:270-4.

23. Kaisemann MC, Kritski AL, Pereira MFC, Trajman A. Dosagem da atividade da adenosina deaminase no líquido pleural para o diagnóstico da tuberculose pleural. J Bras Pneumol. 2004;30(6):549-56.

24. Neves DD, Dias RM, Cunha AlLA, Chibante AMS. Rendimento de variáveis clínicas, radiológicas e laboratoriais para o diagnóstico da tuberculose pleural. J Bras Pneumol. 2004;30(4):409-16.

25. Banales JL, Pineda PR, Fitzgerald JM, Rubio $H$, Selman M, Salazar-Lezama M. Adenosine deaminase in the diagnosis of tuberculous pleural effusion. A report of 218 patients and review of literature. Chest. 1991;99;355-7.

26. Morisson P, Neves DD. Evaluation of adenosine deaminase in the diagnosis of pleural tuberculosis: a Brazilian meta-analysis. J Bras Pneumol. 2008;34(4):217-24.

27. Qiu-Li L, Huan-Zhong S, Ke Wang, Shou-Ming $\mathrm{Q}$, Xue-Jun Q. Diagnostic accuracy of adenosine deaminasa in tuberculous pleurisy: A metaanalysis. Respir Med. 2008;102:744-54.

28. Porcel JM. Adenosine deaminase in nontuberculous lymphocytic pleural effusions. Chest. 2002;121(4):1379-80.

29. Ortiz-Sánchez J. El valor diagnóstico del Test de ADA [tesis doctoral]. Facultad de Medicina, Universidad Nacional Mayor de San Marcos. Lima, Perú. 2002.

30.Segura-Nuñez PR, Sánchez-Sevillano RM. Adenosina deaminasa: La utilidad de su determinación en el líquido pleural para el diagnóstico de pleuresía tuberculosa (19982001) [tesis doctoral]. Facultad de Medicina, Universidad Nacional Mayor de San Marcos. Lima, Perú. 2002.

31. Ibañez-Pradas V, Modesto-Alapont. MBE en Cirugía Pediátrica. Lectura crítica de artículos. Pruebas diagnósticas (I). Cir Pediatr. 2006;19:123-9.

32. Porcel JM. Tuberculous pleural effusion. Lung. 2009;187(5):263-70.

Manuscrito recibido el 21 de enero de 2010 y aceptado para publicación el 16 de marzo de 2010.

\section{Corres3pondencia:}

Jhonatan Boris Quiñones-Silva

San Bartolomé II Etapa Mz A- L26

Huacho, Perú.

Correo-e: jquinones@med-unjfsc.edu.pe 